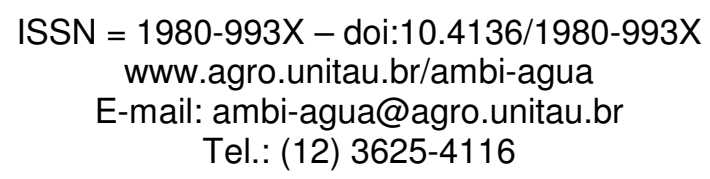

\title{
Uso da Reflectometria no Domínio do Tempo para avaliar a distribuição de nitrato em colunas de solos fertirrigados
} (doi:10.4136/ambi-agua.103)

\author{
Marcelo Rocha dos Santos; Mauro Aparecido Martinez; Antonio Teixeira de Matos; \\ Rubens Alves de Oliveira; João Henrique Zonta \\ DEA - Departamento de Engenharia Agrícola - UFV \\ E-mail: marcelo.rocha@ufv.br; mmauro@ufv.br; atmatos@ufv.br; rubens@ufv.br; joaozonta@hotmail.com
}

\section{RESUMO}

O monitoramento da variação espacial e temporal do teor de água $(\theta)$, da condutividade elétrica do solo $\left(\mathrm{CE}_{\mathrm{s}}\right)$, da condutividade elétrica da solução do solo $\left(\mathrm{CE}_{\mathrm{ss}}\right)$ e da concentração iônica $\left(\mathrm{C}_{\mathrm{i}}\right)$ possibilita estimar a perda de água por percolação profunda, a evapotranspiração da cultura, determinar as regiões de extração de nutrientes pelas plantas e as possíveis perdas de nutrientes por lixiviação. Objetivou-se com esse trabalho avaliar, usando a técnica da TDR, a distribuição de nitrato em colunas de Neossolo Quartzarênico (RQ) e Latossolo Vermelho (LV) fertirrigadas com nitrato de cálcio. Foram realizadas fertirrigações com nitrato de cálcio nas colunas e determinados os teores de água $(\theta)$ e condutividades elétricas do solo $\left(\mathrm{CE}_{\mathrm{s}}\right)$ com uso da TDR. A concentração do nitrato foi estimada a partir de valores de $\theta$ e de $\mathrm{CE}_{\mathrm{s}}$. No RQ, o nitrato alcançou as profundidades de 30,21 e $12 \mathrm{~cm}$ quando se realizaram as fertirrigações no período inicial, central e final da irrigação, respectivamente. No LV, independente do momento da fertirrigação, a maioria do nitrato aplicado ficou adsorvido na camada de 0 a 12 $\mathrm{cm}$ de profundidade.

Palavras-chave: Fertirrigação; Dinâmica de nitrato; TDR.

\section{Use of the Time Domain Reflectometry to evaluate the nitrate distribution in fertirrigated soils columns}

\begin{abstract}
The monitoring of spatial and temporal variation of the soil water content $(\theta)$, of the soil bulk electric conductivity $\left(\mathrm{CE}_{\mathrm{s}}\right)$, of the soil solution electric conductivity $\left(\mathrm{CE}_{\mathrm{ss}}\right)$ and of the ion concentration $\left(\mathrm{C}_{\mathrm{i}}\right)$ enables to estimate the water loss by deep percolation and evapotranspiration, to determine the areas of nutrients extraction by plants and the possible nutrients loss by leaching. The of objective this work was to evaluate, using the TDR technique, the nitrate distribution in Typic Quartzipsamment (RQ) and Oxisol (LV) soil columns fertirrigated with calcium nitrate. Nitrate fertirrigations were accomplished in the columns, and the soil water content $(\theta)$ and the soil bulk electric conductivity $\left(\mathrm{CE}_{\mathrm{s}}\right)$ obtained with TDR. The nitrate concentration was estimated using the values of $\theta$ and of $\mathrm{CE}_{\mathrm{s}}$. In the RQ soil, the nitrate reached 30, 21 e $12 \mathrm{~cm}$ when the fertirrigations were done at the beginning, at the middle and at the end of the irrigation period, respectively. In the LV soil, independently of the moment of the fertirrigation, the nitrate was retained in the layer from 0 to $12 \mathrm{~cm}$ deep.
\end{abstract}

Keywords: Fertirrigation; Nitrate movement; TDR. 


\section{INTRODUÇÃO}

Atualmente no Brasil, tem-se percebido um amplo avanço no uso da irrigação localizada, principalmente em regiões semiáridas, o que tem possibilitado o uso racional da água e a prática da fertirrigação. O emprego dessa técnica tem possibilitado a otimização do uso de insumos em diferentes culturas irrigadas, tanto em aspectos relacionados à produtividade quanto à qualidade dos produtos obtidos (Oliveira e Villas Boas, 2008), principalmente por apresentar características tais como o uso mais eficiente dos nutrientes pelas culturas, a possibilidade em parcelar de forma mais adequada as doses de nutrientes, a redução da mão de obra, a melhoria na conservação do solo, a obtenção de plantas nutridas mais adequadamente, além de acrescentar pouco no custo de um sistema de irrigação.

Entre os nutrientes mais usados na fertirrigação distingue-se o nitrogênio, pelo motivo de grande demanda pelas plantas, pela alta mobilidade no solo, pela disponibilidade de diversas fontes solúveis em água (Ribeiro et al., 1999), além de ser um dos elementos minerais mais limitante ao crescimento vegetal.

O nitrogênio é o nutriente mais aplicado em fertirrigação (Souza et al., 2007a), sendo o seu parcelamento recomendado em razão da sua alta mobilidade no solo, do alto índice salino dos adubos que o contém e da baixa exigência inicial das culturas.

Nos solos de regiões tropicais, a predominância de cargas negativas e a baixa interação química do $\mathrm{NO}_{3}{ }^{-}$com os minerais do solo fazem com que este anion esteja sujeito à lixiviação para camadas mais profundas, podendo atingir o lençol freático com potencial de se tornar um contaminante do ambiente (Cantarella et al., 2007). Em muitas partes do planeta, as águas subterrâneas acham-se impróprias para o consumo humano devido à alta concentração de $\mathrm{NO}_{3}{ }^{-}$, o que está associado a doses excessivas de fertilizantes que têm sido utilizadas na agricultura (Reichardt e Timm, 2004).

Assim, estudos direcionados para o monitoramento dos solutos no solo sob áreas fertirrigadas são de fundamental importância para a tomada de medidas que minimizem problemas relativos a impactos ambientais. Ainda, com a crescente utilização de águas residuárias aplicadas ao solo nas mais diversas formas, a possibilidade de monitoramento da translocação dos íons no solo, tanto no tempo quanto no espaço é de fundamental importância para a prevenção de possíveis impactos ambientais danosos ao lençol freático.

Sob áreas fertirrigadas, o monitoramento da variação espacial e temporal do teor de água $(\theta)$, da condutividade elétrica do solo $\left(\mathrm{CE}_{\mathrm{s}}\right)$, da condutividade elétrica da solução do solo $\left(\mathrm{CE}_{\mathrm{ss}}\right)$ e da concentração iônica $\left(\mathrm{C}_{\mathrm{i}}\right)$, de acordo com (Santana et al., 2007), possibilita estimar a perda de água por percolação profunda, a evapotranspiração da cultura, determinar as regiões de extração de nutrientes pelas plantas e as possíveis perdas de nutrientes por lixiviação.

O monitoramento de íons no solo é geralmente realizado com a utilização de extrator de solução, realização de amostragem do solo ou o uso de extrato da pasta saturada do solo. $\mathrm{O}$ extrator é bastante popular entre os pesquisadores por apresentar resultados precisos a um custo relativamente baixo, porém, sua instalação no solo reduz a precisão dos resultados, pois altera o local em estudo, não permitindo repetições, e limita a obtenção de resultados a um curto espaço de tempo (Souza et al., 2006). A amostragem do solo requer maior tempo para análise e é um processo destrutivo. O extrato da pasta saturada não representa o estado iônico do solo em tempo real. Uma técnica que tem sido utilizada para a avaliação do movimento de solutos no solo é a reflectometria no domínio do tempo (TDR) (Bejat et al., 2000; Mmolawa e Or, 2000), que permite o monitoramento de íons em tempo real, de forma contínua e precisa, mantendo-se a estrutura do solo.

A TDR tornou-se uma importante ferramenta para a medida do conteúdo de água no solo $(\theta)$ e da condutividade elétrica do solo (CEs). O princípio da medida da umidade do solo pela 
técnica da TDR foi pioneiramente introduzida por Davis e Chudobiak (1975) e implementado e validado por Topp et al. (1980). O instrumento TDR funciona emitindo um sinal eletromagnético de alta frequência ao longo de uma sonda inserida no solo. O sinal é refletido no final da sonda e o tempo de viagem do sinal é medido em função do formato de onda resultante, sendo esse tempo relacionado à constante dielétrica do solo $(\mathrm{Ka})$, a qual pode ser relacionada $\operatorname{com} \theta$. Adicionalmente, a atenuação do sinal refletido pode ser relacionado a CEs.

A constante dielétrica medida para um solo é uma média ponderada das constantes dielétricas das frações dos componentes do solo, ou seja, das fases sólida, líquida e gasosa. Como a constante dielétrica da água é de aproximadamente 80, do ar 1 e do material sólido (mineral e orgânico) de 3 a 7, uma pequena variação na umidade volumétrica do solo (q) causará uma mudança considerável da constante dielétrica. Por meio de uma correlação experimental entre a constante dielétrica medida com o TDR e a umidade volumétrica do solo medida por gravimetria, pode-se, portanto, utilizar a técnica de TDR para a determinação da umidade do solo (Topp et al., 1980).

Os primeiros trabalhos que avaliaram as alterações na constante dielétrica do solo, em função do conteúdo de água, identificaram uma relação entre essas variáveis. Davis e Annan (1977) verificaram que havia uma forte dependência da constante dielétrica em relação ao conteúdo de água maior que em relação à composição e à densidade do solo. Topp et al. (1980), estudando cinco tipos de solo, confirmaram, posteriormente, que 93\% das variações da constante dielétrica era devido ao conteúdo de água do solo.

Posteriormente, essa técnica foi utilizada com sucesso em várias pesquisas, objetivandose a determinação da $\theta$ e CEa (Vogeler et al., 1996; Heimovaara et al., 1995; Persson e Uvo, 2003), a modelagem da dinâmica de água e solutos iônicos na zona radicular (Mmolawa e Or, 2000), a identificação do perfil de extração de água pelo sistema radicular das culturas (Coelho e Or, 1996) e a estimativa da evapotranspiração das culturas (Ward e Dunin, 2001). Yu (1998) analisou a aplicação da técnica TDR no transporte experimental de solutos no solo. Shen (1999) utilizou o TDR para a determinação do teor de umidade e o monitoramento da concentração de efluentes durante o fluxo saturado e não saturado através do solo. Segundo De Assis (2008), uma das grandes potencialidades da técnica TDR, além da medida da constante dielétrica, é a medida da condutividade elétrica volumétrica, conforme demonstrado por Topp et al. (1988), Nadler et al. (1991), Dalton (1992), Mojid et al. (1997).

A reflectometria no domínio do tempo fornece a medida da condutividade elétrica volumétrica por meio da mudança da impedância demonstrada pela forma de onda. A impedância final é medida avaliando tempo de viagem ao longo do sinal, em que múltiplas reflexões são resultadas ao fim da sonda. Giese e Tiemann (1975) foram os primeiros a determinarem a condutividade elétrica volumétrica $\left(\mathrm{EC}_{\mathrm{b}}\right)$ por meio da análise da forma de onda utilizando a técnica TDR.

Para o uso dessa metodologia, é necessária a confecção de uma curva de calibração para cada tipo de solo, sendo considerado um inconveniente normalmente aceitável comparado com as vantagens que apresenta em relação a outras técnicas. Diversos trabalhos referentes a calibração de sondas de TDR são encontrados na literatura, como os de Trintinalha et al. (2004), Villwock et al. (2004), Coelho et al. (2006) entre outros.

As vantagens inerentes da técnica da TDR, como a precisão, o fato de ser um método não destrutivo, a não-utilização de radiação ionizante e a possibilidade de automação e de acoplamento a dispositivos multiplicadores de leituras são justificativas fortes para a adoção cada vez maior da técnica em substituição a outros métodos (Topp et al., 1980; Coelho e Or, 1996).

Com a utilização da TDR, pode-se, ao mesmo tempo, determinar num dado local do solo, o teor de água e a condutividade elétrica do solo $\left(\mathrm{CE}_{\mathrm{s}}\right)$, o que abre caminho para inferir a condutividade elétrica da solução do solo $\left(\mathrm{CE}_{\mathrm{ss}}\right)$ e a concentração de um dado nutriente 
aplicado $\left(\mathrm{C}_{\mathrm{i}}\right)$. A $\mathrm{CE}_{\mathrm{s}}$, determinada com a utilização da TDR, é variável conforme o teor de água no solo $(\theta)$, uma vez que leva em conta a $\mathrm{CE}_{\mathrm{ss}}$ e a condutividade elétrica da fração sólida do solo $\left(\mathrm{CE}_{\mathrm{fs}}\right)$.

Vários modelos matemáticos têm sido usados para estimar $\mathrm{CE}_{\mathrm{s}}$ em função de $\theta$ e da $\mathrm{CE}_{\mathrm{ss}}$, os mais usuais têm sido o de Rhoades et al. (1976); Nadler et al. (1984); Heimovaara et al. (1995); Rhoades et al. (1989); Vogeler et al. (1996); Mualen E Friedman (1991). Os modelos de Rhoades et al. (1976) e o de Vogeler et al. (1996) são os que têm apresentado melhores resultados (Santos, 2008; Santana et al., 2007).

Objetivou-se com este trabalho avaliar-se em laboratório, usando a técnica da TDR, a distribuição de nitrato em colunas de Neossolo Quartzarênico e de Latossolo Vermelho fertirrigadas com nitrato de cálcio em diferentes momentos durante a irrigação.

\section{MATERIAL E MÉTODOS}

O experimento foi conduzido em colunas de Neossolo Quartzarênico (RQ) e Latossolo Vermelho (LV) montadas em laboratório, no Departamento de Engenharia Agrícola da Universidade Federal de Viçosa - UFV.

O solo do tipo RQ foi coletado nos primeiros $40 \mathrm{~cm}$ de profundidade, nas proximidades da Fazenda Experimental da EPAMIG à latitude de $15^{\circ} 32^{\prime} \mathrm{S}$ e longitude de $43^{\circ} 46^{\prime} \mathrm{W}$, em Mocambinho, no município de Jaíba, MG. O LV foi coletado na região do Alto Paranaíba, MG à latitude de $19^{\circ} 21^{\prime} \mathrm{S}$ e longitude de $046^{\circ} 07^{\prime} \mathrm{W}$, na camada entre 50 e $120 \mathrm{~cm}$ de profundidade, a qual corresponderia aos horizontes Bw1 e Bw2 (Rolim Neto, 2002). O solo RQ foi escolhido por apresentar textura arenosa e o LV por apresentar característica de eletropositividade. Os solos coletados foram secos ao ar e passados em peneira com malha de 2,0 mm. Amostras destes foram utilizadas para determinação das características físicohídricas e químicas, apresentadas na Tabela 1.

Para monitoramento do teor de água no solo $(\theta)$ e da condutividade elétrica do solo $\left(\mathrm{CE}_{\mathrm{s}}\right)$ foram utilizadas sondas de TDR confeccionadas em laboratório, sendo estas compostas de três hastes de $0,13 \mathrm{~m}$ dos quais com $0,10 \mathrm{~m}$ de comprimento efetivo e $0,03 \mathrm{~m}$ de comprimento coberto por resina, espaçadas entre si de $1,7 \mathrm{~cm}$ e sem resistor na haste central. As sondas de TDR estavam conectadas em multiplexadores SDMX-50, sendo este conectado a um equipamento modelo TDR 100, que enviava os dados para um datalogger modelo CR10X, todos equipamentos da fabricante Campbell Scientific, Inc.

As colunas foram confeccionadas com tubos de PVC de $150 \mathrm{~mm}$ de diâmetro e $0,70 \mathrm{~m}$ de comprimento, fechados em uma das extremidades, com um dreno na base, preenchidas com uma camada de material de solo de $0,66 \mathrm{~m}$, sobrando uma borda de $0,04 \mathrm{~m}$. O solo foi compactado nos recipientes de modo a se obter massas especificas semelhantes às encontradas no campo (Tabela 1). Sondas de TDR foram inseridas horizontalmente nas colunas de solo num espaçamento de 9 centímetros entre si, para possibilitar a quantificação do teor de água e da condutividade elétrica do solo, sendo a primeira sonda inserida numa profundidade de $3 \mathrm{~cm}$ (Figura 1). 
SANTOS, M. R.; MARTINEZ, M. A.; MATOS, A. T.; OLIVEIRA, R. A.; ZONTA, J. H. Uso da Reflectometria no Domínio do Tempo para avaliar a distribuição de nitrato em colunas de solos fertirrigados. Ambi-Agua, Taubaté, v. 4, n. 3, p. 67-81, 2009. (doi:10.4136/ambi-agua.103)

Tabela 1. Características físico-hídricas e químicas do Neossolo Quartzarênico (RQ) e Latossolo Vermelho (LV).

\begin{tabular}{|c|c|c|c|c|c|}
\hline \multicolumn{3}{|c|}{ Características físico-hídricas } & \multicolumn{3}{|c|}{ Características químicas } \\
\hline & RQ & $\mathbf{L V}$ & & RQ & $\mathbf{L V}$ \\
\hline Areia Grossa $\left(\mathrm{kg} \mathrm{kg}^{-1}\right)^{1}$ & 0,63 & 0,02 & pH em água & 5,34 & 5,01 \\
\hline Areia Fina $\left(\mathrm{kg} \mathrm{kg}^{-1}\right)^{1}$ & 0,24 & 0,03 & $\mathrm{pH}$ em KCl & 4,38 & 5,45 \\
\hline Silte $\left(\mathrm{kg} \mathrm{kg}^{-1}\right)^{2}$ & 0,02 & 0,11 & $\mathrm{P}\left(\mathrm{mg} \mathrm{dm} \mathrm{m}^{-3}\right)$ & 2,10 & 0,50 \\
\hline Argila $\left(\mathrm{kg} \mathrm{kg}^{-1}\right)^{2}$ & 0,11 & 0,84 & $\mathrm{~K}\left(\mathrm{mg} \mathrm{dm}^{-3}\right)$ & 46,0 & 10,0 \\
\hline Massa específica do solo $\left(\mathrm{kg} \mathrm{dm}^{-3}\right)^{3}$ & 1,49 & 1,07 & $\mathrm{Ca}^{2+}\left(\mathrm{cmol}_{\mathrm{c}} \mathrm{dm}^{-3}\right)$ & 0,76 & 0,09 \\
\hline Massa especifica das partículas $\left(\mathrm{kg} \mathrm{dm}^{-3}\right)^{4}$ & 2,73 & 2,53 & $\mathrm{Mg}^{2+}\left(\mathrm{cmol}_{\mathrm{c}} \mathrm{dm}^{-3}\right)$ & 0,10 & 0,00 \\
\hline Retenção de água a $-10 \mathrm{kPa}\left(\mathrm{cm}^{3} \mathrm{~cm}^{-3}\right)^{5}$ & 0,09 & 0,39 & $\mathrm{Al}^{3+}\left(\mathrm{cmol}_{\mathrm{c}} \mathrm{dm}^{-3}\right)$ & 0,19 & 0,00 \\
\hline Retenção de água a $-1.500 \mathrm{kPa}\left(\mathrm{m}^{3} \mathrm{~m}^{-3}\right)^{5}$ & 0,05 & 0,29 & $\mathrm{H}+\mathrm{Al}\left(\mathrm{cmol}_{\mathrm{c}} \mathrm{dm}^{-3}\right)$ & 1,10 & 3,00 \\
\hline Porosidade $\left(\mathrm{m}^{3} \mathrm{~m}^{-3}\right)^{6}$ & 0,45 & 0,58 & $\mathrm{SB}\left(\mathrm{cmol}_{\mathrm{c}} \mathrm{dm}^{-3}\right)$ & 0,98 & 0,12 \\
\hline Macroporosidade $\left(\mathrm{m}^{3} \mathrm{~m}^{-3}\right)^{7}$ & 0,36 & 0,16 & CTC $(\mathrm{t})\left(\mathrm{cmol}_{\mathrm{c}} \mathrm{dm}^{-3}\right)$ & 1,17 & 0,12 \\
\hline Microporosidade $\left(\mathrm{m}^{3} \mathrm{~m}^{-3}\right)^{8}$ & 0,09 & 0,42 & $\mathrm{CTC}(\mathrm{T})\left(\mathrm{cmol}_{\mathrm{c}} \mathrm{dm}^{-3}\right)$ & 2,08 & 3,12 \\
\hline \multirow[t]{4}{*}{ Condutividade Hidráulica $\left(\mathrm{cm} \mathrm{h}^{-1}\right)^{9}$} & 52,81 & 28,35 & $\mathrm{~V}(\%)$ & 47,10 & 3,80 \\
\hline & & & $\mathrm{m}(\%)$ & 16,20 & 0,00 \\
\hline & & & Matéria orgânica (dag kg-1) & 0,78 & 1,94 \\
\hline & & & P-rem $\left(\mathrm{mg} \mathrm{L}^{-1}\right)$ & 41,9 & 3,6 \\
\hline & \multicolumn{3}{|c|}{$\mathrm{P}-\mathrm{Na}-\mathrm{K}-$ Extrator Mehlich 1} \\
\hline \multirow{2}{*}{\multicolumn{3}{|c|}{${ }^{1}$ Por peneiramento }} & \multicolumn{3}{|c|}{$\mathrm{Ca}-\mathrm{Mg}-\mathrm{Al}-$ Extrator: $\mathrm{KCl}-1 \mathrm{~mol} \cdot \mathrm{L}^{-1}$} \\
\hline & & & \multirow{2}{*}{\multicolumn{3}{|c|}{$\begin{array}{l}\mathrm{H}+\mathrm{Al}-\text { Extrator Acetato de Cálcio } 0,5 \mathrm{~mol} / \mathrm{L}^{-1}- \\
\mathrm{pH} 7,0\end{array}$}} \\
\hline \multicolumn{3}{|c|}{${ }^{3}$ Método da proveta e anel volumétrico para S1 e S2 } & & & \\
\hline \multicolumn{3}{|l|}{ respectivamente } & \multicolumn{3}{|l|}{$\mathrm{SB}=$ Soma de Bases } \\
\hline \multicolumn{3}{|l|}{${ }^{4}$ Método do balão volumétrico } & \multicolumn{3}{|c|}{ CTC $(\mathrm{t})$ - Capacidade de Troca Catiônica Efetiva } \\
\hline \multicolumn{3}{|l|}{${ }^{5}$ Extrator de placa porosa } & \multicolumn{3}{|c|}{ CTC (T) - Capacidade de Troca Catiônica a pH } \\
\hline \multicolumn{3}{|l|}{${ }^{6} P T=1-\rho s / \rho p$} & \multicolumn{3}{|c|}{7,0} \\
\hline \multicolumn{3}{|l|}{${ }^{7} \mathrm{P}_{\mathrm{MACRO}}=\mathrm{P}_{\mathrm{TOTAL}}-\mathrm{P}_{\mathrm{MICRO}}$} & \multicolumn{3}{|c|}{$\begin{array}{l}\mathrm{V}=\text { Índice de Saturação de Bases } \\
\mathrm{m}=\text { Índice de Saturacão de Alumínio }\end{array}$} \\
\hline \multirow{2}{*}{\multicolumn{3}{|c|}{$\begin{array}{l}{ }^{8} \text { Mesa de tensão } \\
{ }^{8} \text { Permeâmetro de carga constante }\end{array}$}} & \multirow{2}{*}{\multicolumn{3}{|c|}{$\begin{array}{l}\mathrm{m}=\text { Índice de Saturação de Alumínio } \\
\text { Mat. Org = C. Org x } 1,724-\text { Walkley-Black }\end{array}$}} \\
\hline & & & & & $\begin{array}{l}\text { Mat. Org = C. Org x } 1,724-\text { Walkley-Black } \\
\text { P-rem = Fósforo Remanescente }\end{array}$ \\
\hline
\end{tabular}

Nove colunas foram preenchidas com o RQ e nove com LV, para cada solo, as colunas foram fertirrigadas com nitrato de cálcio. Em cada solo, a fertirrigação foi aplicada no início, no meio e no final do período de irrigação. Foi construído um sistema com frascos de Mariotte conectados a um recipiente gotejador, que possibilitou manter uma carga hidráulica constante, possibilitando a aplicação de água e solução fertilizante numa vazão média de $0,73 \mathrm{~L} \mathrm{~h}^{-1} \mathrm{e}$ ainda, a aplicação independente da irrigação ou fertirrigação.

Após a montagem, as colunas foram submetidas a uma primeira irrigação, visando elevar o teor de água na camada de 0 a $0,40 \mathrm{~m}$ de profundidade a valores correspondentes a capacidade de campo. Terminada essa irrigação, esperou-se um período de três dias para redistribuição da água nas colunas de solo. Cessada a redistribuição, foi feita uma segunda irrigação com duração de, aproximadamente, quarenta minutos na qual foi aplicada uma lâmina de $0,38 \mathrm{~L}$ de água e $0,10 \mathrm{~L}$ de solução fertilizante em cada coluna. A solução fertilizante à concentração de $3,55 \mathrm{~g} \mathrm{~L}^{-1}$ de nitrato de cálcio foi aplicada no inicio, no meio ou no final da irrigação. A dose do fertilizante aplicada, de $33,3 \mathrm{~kg} \mathrm{ha}^{-1} \mathrm{de} \mathrm{N}$, foi baseada em valores recomendados de $400 \mathrm{~kg} \mathrm{ha}^{-1} \mathrm{ano}^{-1}$ de $\mathrm{N}$ para a cultura da bananeira (Borges et al., 2006), distribuídos numa frequência mensal de aplicação. A irrigação foi feita com água destilada e a solução fertilizante (mistura do fertilizante com água destilada) apresentava condutividade elétrica de 3,66 dS m${ }^{-1}$. Após a irrigação, as colunas eram cobertas com plástico para evitar evaporação e permitir somente a redistribuição. 


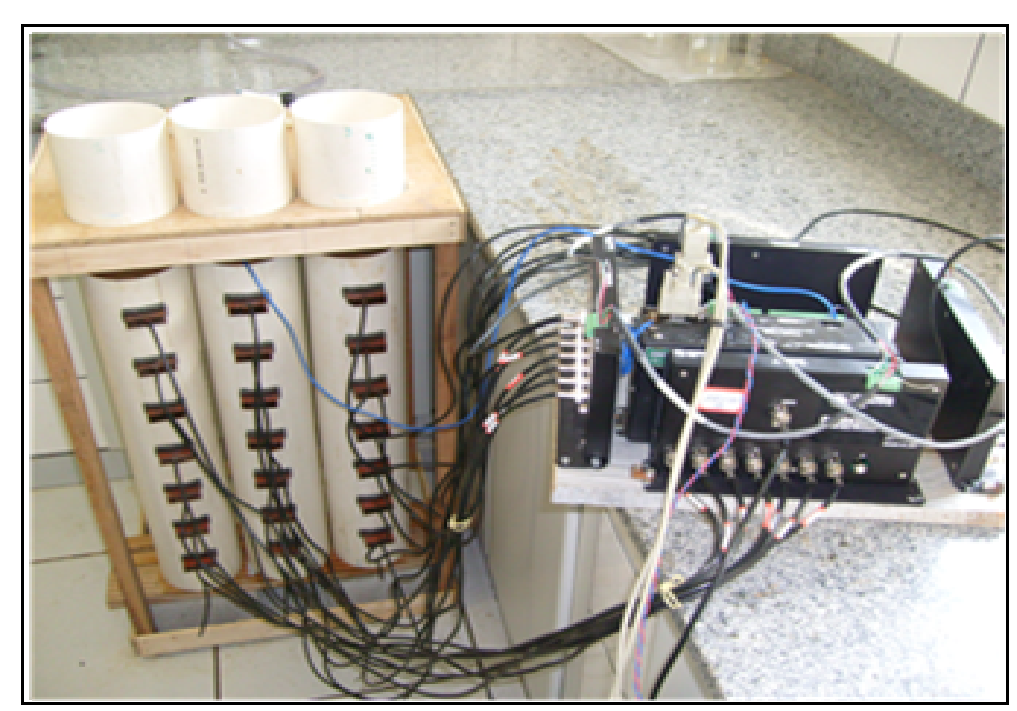

Figura 1. Vista geral das colunas com as sondas e o equipamento de TDR.

Durante o período de irrigação, fertirrigação e de redistribuição, os teores de água e as condutividades elétricas do solo foram determinadas usando um aparelho de TDR conectado a um datalogger. O datalogger foi programado a emitir comandos para a TDR fazer leitura em intervalos de 3 minutos durante a irrigação ou fertirrigação e em intervalo de 10 minutos após o final da irrigação, quando ocorria a redistribuição da água e dos fertilizantes no solo. Cada teste era encerrado quando a variação do teor de água com o tempo era mínima na coluna.

Para determinação do teor de água no solo com o uso da TDR foi utilizado um modelo polinomial cúbico (Equação 1) e um modelo linear (Equação 2) para os solos RQ e LV, respectivamente, conforme Santos (2008).

$$
\begin{aligned}
& \theta_{\mathrm{RQ}}=0,0000437 \mathrm{Ka}^{3}-0,001829 \mathrm{Ka}^{2}+0,036518 \mathrm{Ka}-0,1706486 \\
& \theta_{\mathrm{LV}}=0,01188095 \mathrm{Ka}+0,05948608
\end{aligned}
$$

Em que $\theta_{\mathrm{RQ}}$ e $\theta_{\mathrm{LV}}$ são os teores de água $\left(\mathrm{m}^{3} \mathrm{~m}^{-3}\right)$ nos solos RQ e $\mathrm{LV}$, respectivamente e Ka é a constante dielétrica do solo.

O modelo matemático proposto por Vogeler et al. (1996) e calibrado por Santos (2008) foi usado para estimar a condutividade elétrica da solução do solo $\left(\mathrm{CE}_{\mathrm{ss}}\right)$ em função do teor de água $(\theta)$ e da condutividade elétrica do solo $\left(\mathrm{CE}_{\mathrm{s}}\right)$. Esse modelo foi conjugado com outro que relacionou a $\mathrm{CE}_{\mathrm{ss}}$ com a concentração do nutriente, resultando em um modelo final (Equações 3 e 4) capaz de estimar a concentração de nitrato no LV e RQ, respectivamente.

$$
\begin{aligned}
& C i_{\mathrm{NO}_{3}^{-}}(L V)=\left[\left(\frac{C E_{S}-\left(0,11557 \times \theta_{L V}+0,00452\right)}{\left(0,34053 \times \theta_{L V}+0,00421\right)}\right)-0,014334\right] / 9,00885 \\
& C i_{N O_{3}^{-}}(R Q)=\left[\left(\frac{C E_{S}-\left(0,236741 \times \theta_{R Q}+0,032169\right)}{\left(0,564989 \times \theta_{R Q}+0,008389\right)}\right)+0,14464\right] / 0,05757
\end{aligned}
$$

em que:

$$
\begin{aligned}
& C i_{\mathrm{NO}_{3}^{-}} \text {é a concentração do nitrato }\left(\mathrm{mg} \mathrm{L}^{-1}\right) \\
& \mathrm{CE}_{\mathrm{S}} \text { é a condutividade elétrica do solo }\left(\mathrm{dS} \mathrm{m}^{-1}\right) \mathrm{e}
\end{aligned}
$$




\section{RESULTADOS E DISCUSSÃO}

Os teores de água e as condutividades elétricas do solo RQ, desde a primeira irrigação até o final do tratamento com o nitrato de cálcio aplicado no inicio da segunda irrigação são mostrados na Figura 2. A primeira irrigação, feita para elevar o teor de água na coluna, durou aproximadamente 1,5 horas e a segunda irrigação teve inicio a aproximadamente 88 horas após o término da primeira irrigação, quando a coluna já apresentava em todo o perfil teores de água acima do ponto de murcha permanente. Verifica-se nesse solo (Figura 2), que durante a primeira irrigação e o período de redistribuição, os sais contidos originalmente no solo foram lixiviados, por fluxo de massa, para as camadas mais profundas da coluna.
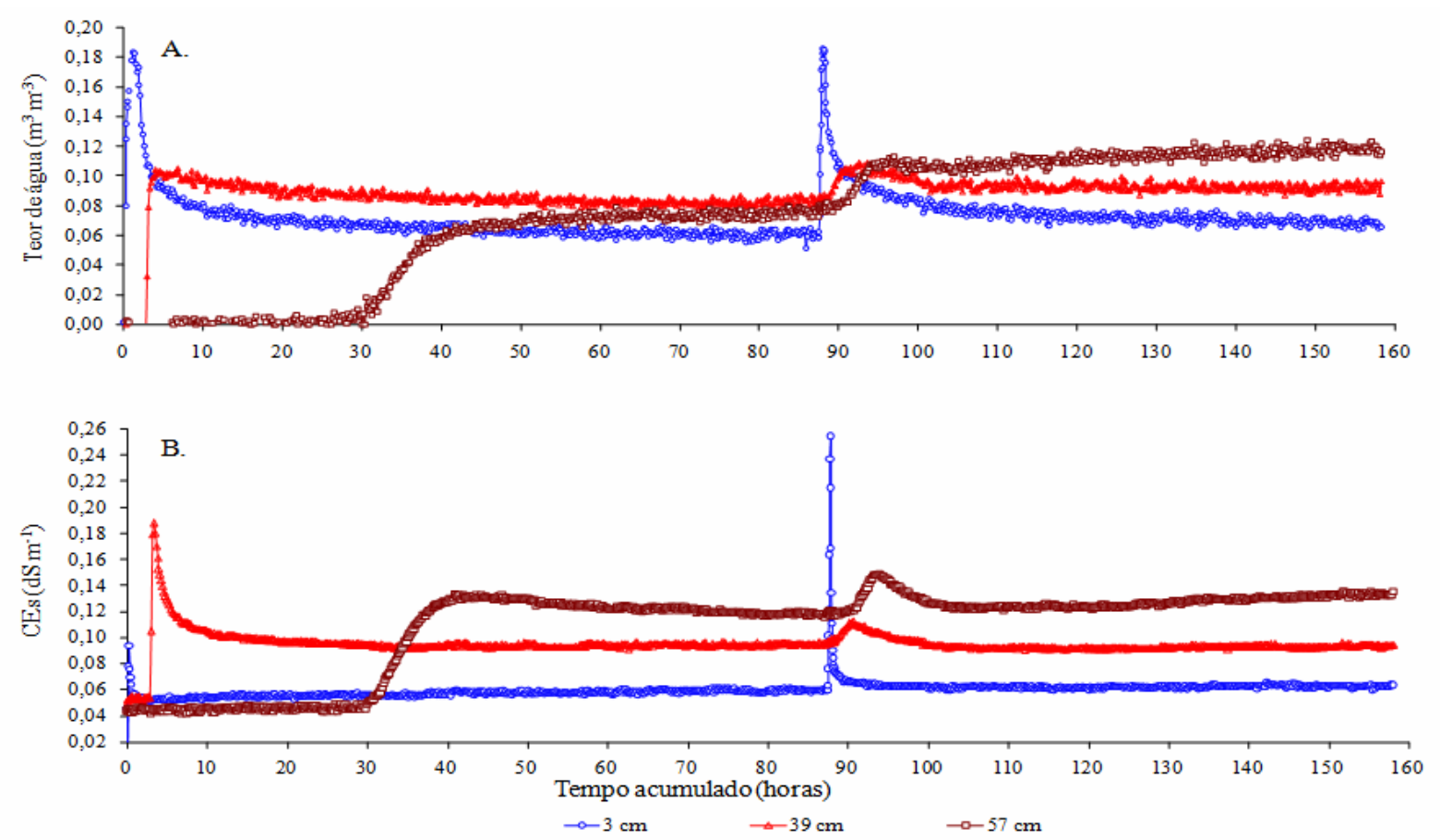

Figura 2. Teor de água (A) e condutividade elétrica (B) no solo RQ, nas profundidades de 3, 39 e $57 \mathrm{~cm}$, a partir do inicio da primeira irrigação com o nitrato de cálcio aplicado na segunda irrigação.

Analisando-se a Figura 3, observa-se a influência do momento da aplicação da solução fertilizante na condutividade elétrica do solo. Nota-se que a condutividade elétrica começa a aumentar a partir dos 30 minutos na profundidade de $12 \mathrm{~cm}$ (Figura 3B), a partir de 1 hora, na profundidade de $12 \mathrm{~cm}$ (Figura 3D) e a partir de 2 horas, na profundidade de $12 \mathrm{~cm}$ (Figura $3 F)$ após o início da irrigação, para fertirrigação no início, meio e final do período de irrigação, respectivamente, indicando que o soluto alcançou essas profundidades nos referidos tempos. 

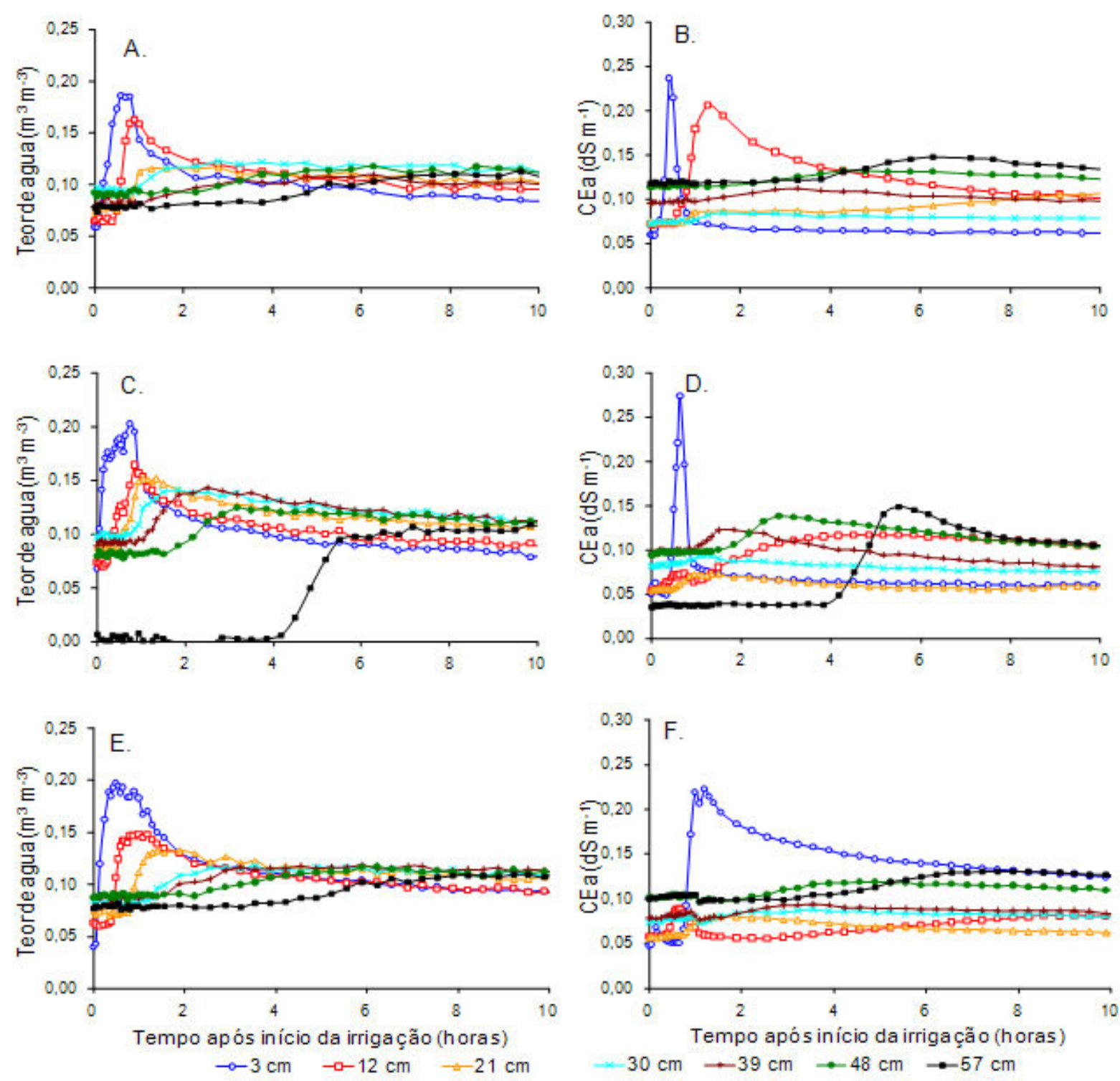

Figura 3. Teor de água e condutividade elétrica do solo RQ em diferentes profundidades, com aplicação de nitrato de cálcio no inicio (A e B), meio (C e D) e final (E e F) da segunda irrigação.

Na Figura 4, está mostrada a distribuição do nitrato no RQ, para os diferentes momentos de aplicação do nitrato de cálcio durante a irrigação. Verifica-se que o nitrato alcança os 30, 21 e $12 \mathrm{~cm}$ de profundidade (Figuras 4A, B e C), quando a aplicação fertilizante foi realizada no início, meio e final da irrigação, respectivamente.

Ainda de acordo com a Figura 4, nota-se que já no início da aplicação da solução fertilizante a concentração do nitrato em cada profundidade apresentava valor maior do que zero, fato ocasionado provavelmente pelo arrastamento de sais das camadas superficiais para as mais profundas durante a primeira irrigação. 


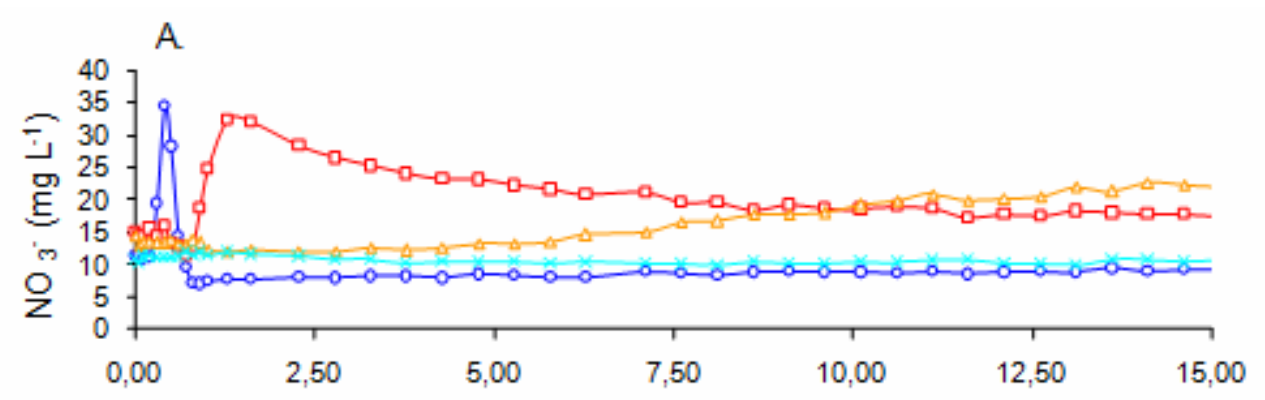

B.
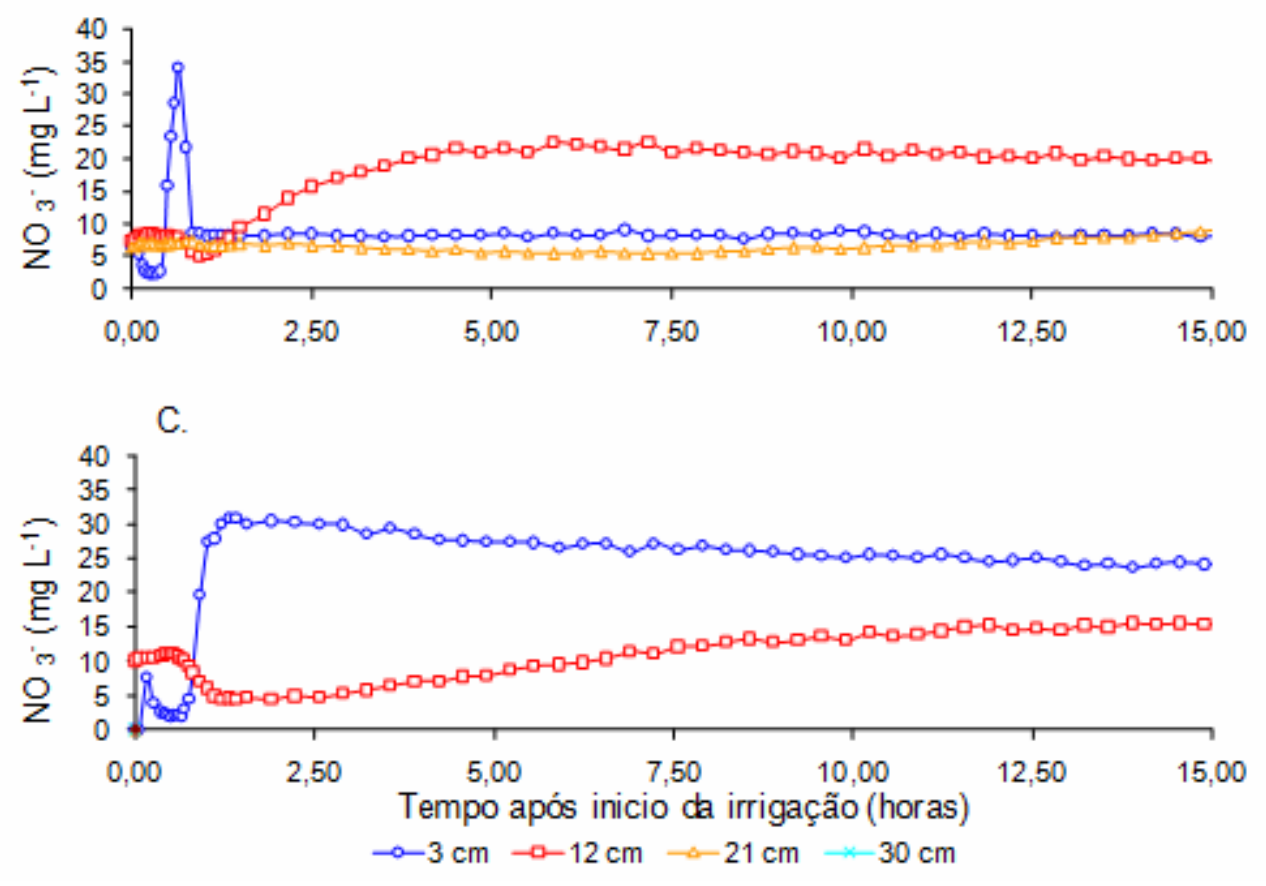

Figura 4. Teor de nitrato na solução do solo RQ com fertirrigação realizada no inicio (A), meio (B) e final (C) da irrigação.

Com esses resultados é possível supor uma perda por lixiviação além da região correspondente à zona de absorção radicular, para culturas que possuam sistema radicular pouco profundo, após sucessivas irrigações, quando aplicado o nitrato de cálcio em RQ em condições práticas de campo. O presente estudo teve como base uma aplicação de nitrato de cálcio numa dose de $400 \mathrm{~kg} \mathrm{ha}^{-1} \mathrm{ano}^{-1}$ de $\mathrm{N}$ numa frequência de aplicação mensal. É de se esperar que a aplicação a maiores frequências apresente adsorção em menores profundidades, uma vez que haverá redução na quantidade aplicada e na concentração, havendo menor saturação de sítios de adsorção de $\mathrm{NO}_{3}{ }^{-}$no solo. Vale ainda ressaltar que aqui não houve extração por parte do sistema radicular das culturas.

Verifica-se na Figura 5 a influência do momento da aplicação da solução fertilizante durante a irrigação na distribuição da água e da condutividade elétrica no LV, no qual se observa o retardo do avanço do sal pelos valores de $\mathrm{CE}_{\mathrm{s}}$ em relação à distribuição do teor de água. Sendo que a $\mathrm{CE}_{\mathrm{s}}$ é alterada apenas a $3 \mathrm{~cm}$ para todos os casos e em 12, para fertirrigação no inicio, enquanto, a água, alcança pelo menos os $48 \mathrm{~cm}$ de profundidade. 

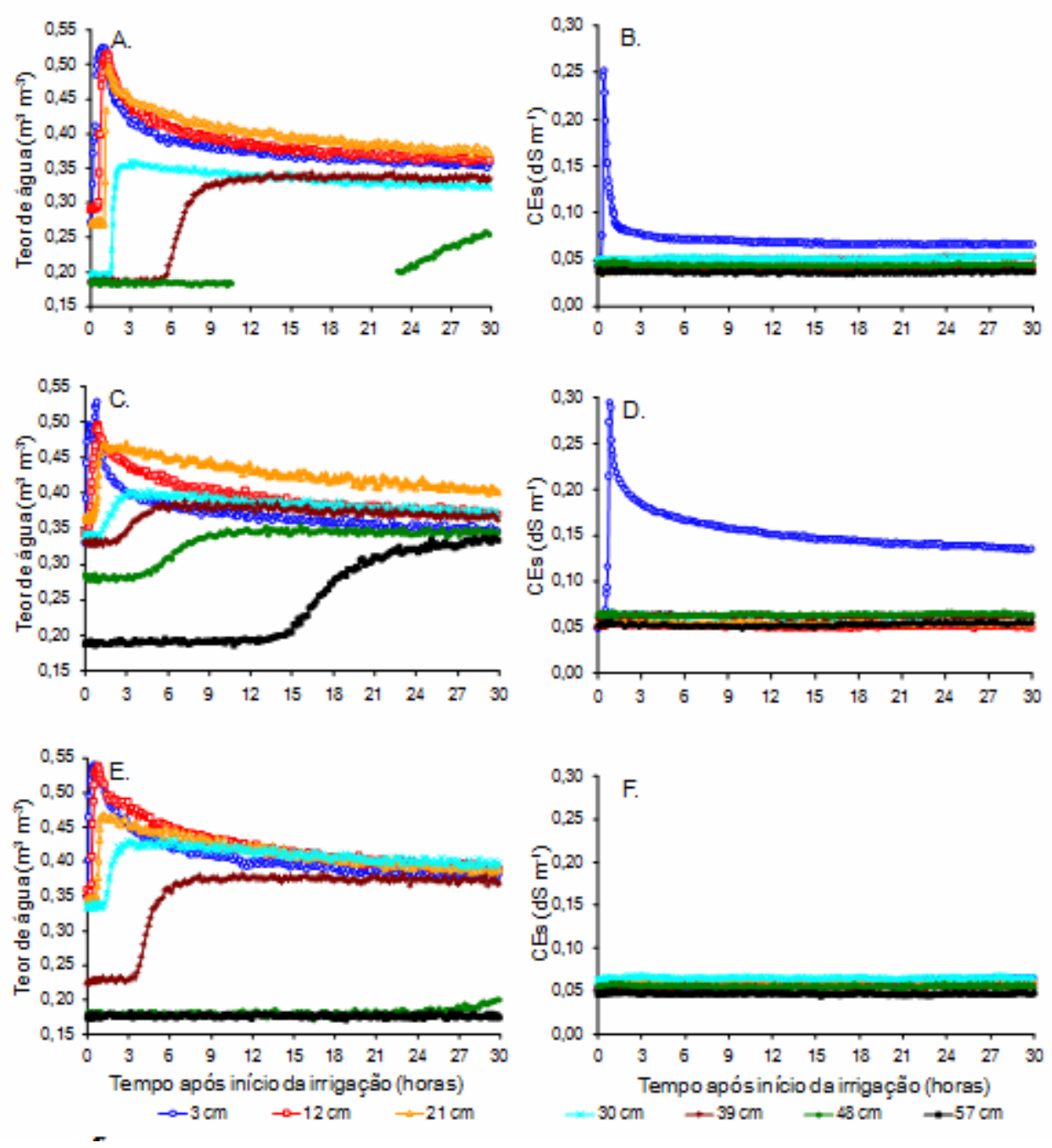

Figura 5. Teor de água e condutividade elétrica do LV com aplicação de nitrato de cálcio no inicio (A e B), meio (C e D) e final (E e F) da segunda irrigação.

Analisando a Figura 6, verifica-se que o nitrato avançou além dos $3 \mathrm{~cm}$ de profundidade, alcançando os $12 \mathrm{~cm}$, quando a aplicação do nitrato de cálcio foi realizada no inicio da irrigação (Figura 6 A.). Quando a fertirrigação foi realizada no período central da irrigação, o nitrato passa abaixo da profundidade de $3 \mathrm{~cm}$ mas não atingiu a profundidade de $12 \mathrm{~cm}$, ficando grande parte retido na camada de 0 a $3 \mathrm{~cm}$ de profundidade (Figura 6B.). No caso da fertirrigação realizada no final do período de irrigação, observa-se que o nitrato fica adsorvido na camada superficial (Figura 6C.), onde a partir de oito horas após o inicio da irrigação, este começa a chegar aos três centímetros de profundidade.

Souza et al. (2007b) trabalhando com dinâmica de nitrato em Latossolo VermelhoAmarelo sob 7 fertirrigações consecutivas, monitoradas com uso da TDR, verificaram que para uma aplicação de $\mathrm{KNO}_{3}$, numa concentração de $14,5 \mathrm{mmol} \mathrm{L}{ }^{-1}$, o sal alcançou uma profundidade de, aproximadamente, 0,22 $\mathrm{m}$ e o maior armazenamento ocorreu na camada de 0-0,10 m durante todas as aplicações da solução no solo. Em média, 53\% da solução aplicada foi armazenada nessa camada, e $28 \%$ na camada de 0,10-0,20 m. O movimento da solução para a terceira camada $(0,20-0,30 \mathrm{~m})$ foi observado apenas após a $2^{\mathrm{a}}$ aplicação, atingindo a última camada $(0,30-0,40 \mathrm{~m})$ após a $4^{\mathrm{a}}$ irrigação. Porém, para o latossolo utilizado nesse trabalho, praticamente todo nitrato ficou adsorvido na camada superficial inferior a $12 \mathrm{~cm}$. 

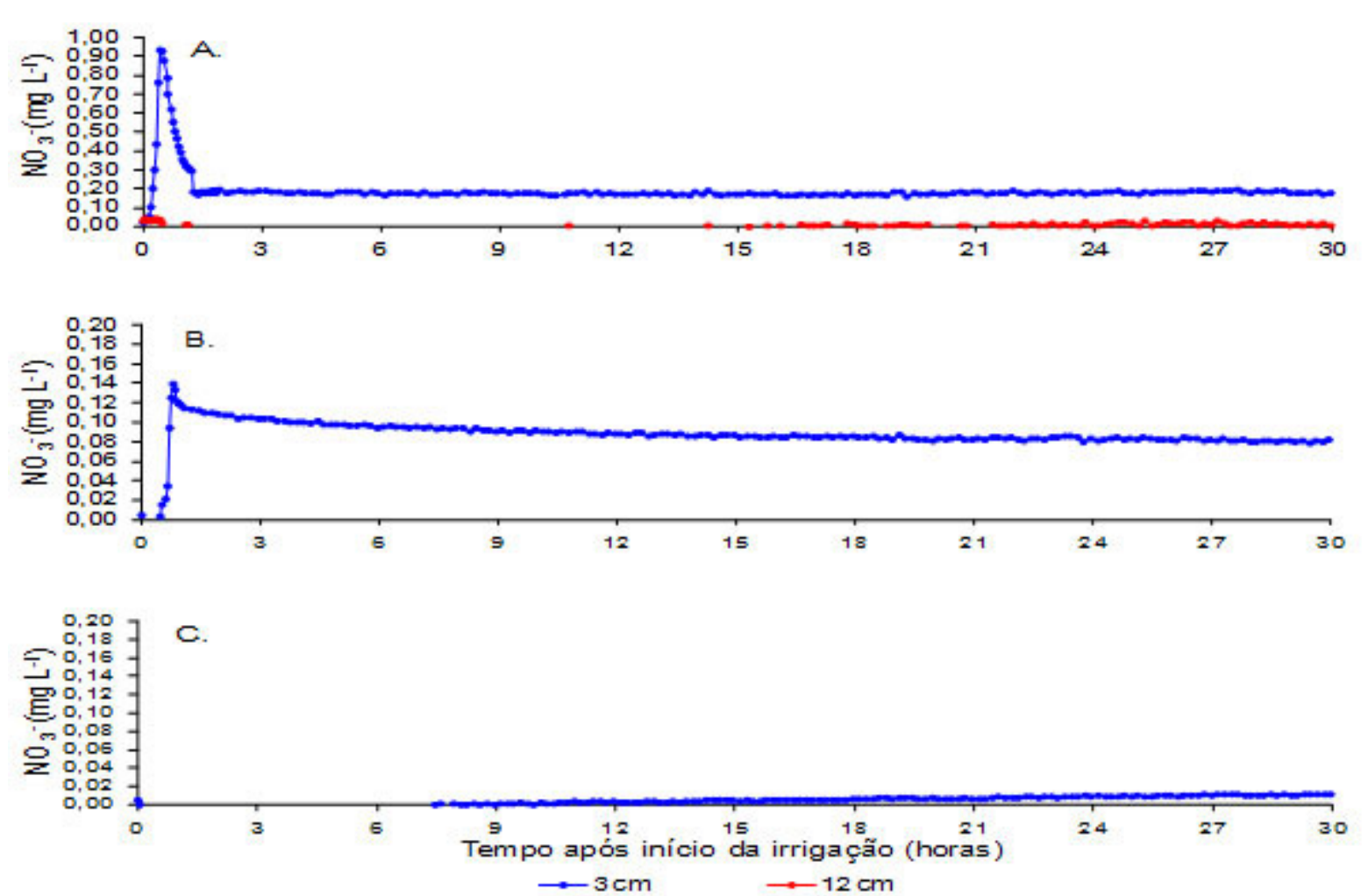

Figura 6. Teor de nitrato no LV com fertirrigação realizada no inicio (A), meio (B) e final (C) da irrigação.

Os resultados obtidos nesse trabalho para o LV apresentam menor avanço do nitrato no solo, se comparados com aqueles obtidos por Souza et al. (2007b), considerando-se apenas uma irrigação. Torna-se importante ressaltar que pelo fato de o latossolo usado neste trabalho possuir característica de eletropositividade, contribui com maiores sítios de adsorção do nitrato, fazendo com que o nitrato fique adsorvido à camada superficial.

Comparando-se a distribuição do nitrato nos solos em estudo, verifica-se a influência das características físicas e químicas do solo na adsorção deste nutriente. Como o LV apresenta característica de eletropositividade, este possui disponíveis muitos sítios de adsorção para o nitrato, resultando nessa diferença de mobilidade, concentração e distribuição entre os dois solos em estudo. Oliveira et al. (2000), estudando a adsorção de nitrato em Latossolo de cerrado, verificaram que a maior adsorção de nitrato ocorre na subsuperfície do solo, em razão da inversão de cargas. Gomes et al. (2004), ao comparar a distribuição de nitrato em solos de textura argilosa e arenosa, constatou que ocorre uma maior sorção de nitrato nos solos de textura argilosa, sendo o solo arenoso aquele que apresentou a maior lixiviação de sais totais e nitrato, independente das dosagens aplicadas.

Lelis Neto (2008), trabalhando com aplicação de vinhaça em diferentes tipos de solo, observou que o íon nitrato ficou mais fortemente retido no solo argiloso se comparado ao solo arenoso, concluindo que a percolação do íon nitrato está diretamente relacionada com o tipo de solo. Segundo Muchovej e Rechcigl, (1994), a textura do solo e o conteúdo de matéria orgânica são os fatores de maior influência nas perdas por lixiviação, e os solos de textura mais grosseira e de baixo conteúdo de matéria orgânica tendem a permitir maiores perdas de nitrato por lixiviação, enquanto as menores perdas devem ocorrer em solos argilosos, corroborando com os resultados obtidos neste presente trabalho.

Com isso, podemos observar que, no caso de se utilizar a fertirrigação como meio de fornecer o nitrogênio $(\mathrm{N})$ as plantas, como por exemplo, com uso de águas residuárias ricas 
em nitrogênio, em um programa de adubação para os solos em estudo, haveria a necessidade de se determinar o momento ótimo de aplicação do nutriente e a lâmina de água aplicada, de modo que, após sua aplicação, a irrigação continuaria com o objetivo de transportar o nitrato até a profundidade em que se encontra o maior volume de raízes ativas (Coelho, 1994).

\section{CONCLUSÕES}

1. A técnica TDR, em conjunto com uso de modelos ajustados, demonstrou-se hábil para o monitoramento do transporte de solutos no solo, independente do tipo de solo em uso e da dosagem do nutriente aplicada.

2. O momento da fertirrigação influenciou fortemente a distribuição de nitrato no Neossolo Quartzarênico, atingindo as profundidades de 30, 21 e $12 \mathrm{~cm}$ quando realizada no inicio, meio e final do período de irrigação, respectivamente.

3. A distribuição do nitrato no Latossolo Vermelho foi pouco influenciada pelo momento da realização da fertirrigação, ficando a maioria do nitrato aplicado retido na camada de 0 a 3 $\mathrm{cm}$ de profundidade.

4. Maiores cuidados devem ser tomados na aplicação de fertirrigação com águas ricas em nitrogênio, como é o caso de águas residuárias, quando se trabalha em solos de textura arenosa.

\section{REFERENCIAS}

BEJAT, L.; PERFECT, E.; QUINSENBERRY, V. L.; COYNE, M. S.; HASZLER, G. R. Solute transport as related to soil structure in unsaturated intact soil blocks. Soil Science Society of America Journal, v. 64, n. 3, p. 818-26, 2000.

BORGES, A. L.; COELHO, E. F.; COSTA, E. L.; SILVA, J. T. A. Fertirrigação da bananeira. Cruz das Almas: EMBRAPA-CNPMF, 2006. 8p. (Circular Técnica, 84)

CANTARELLA, H. Nitrogênio. IN: NOVAIS, R. F.; ALVAREZ V, V. H.; BARROS, N. F.; FONTES, R. L. F.; CANTARUTTI, R. B.; NEVES, J. C. L. (eds.). Fertilidade do solo. Viçosa: MG/SBCS, 2007.v.1, p. 375-470.

COELHO, A. M. Fertirrigação. In: COSTA, E. F.; VIEIRA, R. F.; VIANA, P. A. Quimigação: aplicação de produtos químicos e biológicos via irrigação. Brasília: EMBRAPA-SPI, 1994. 315 p.

COELHO, E. F.; OR, D. Flow and uptake patterns affecting soil water sensor placement for drip irrigation management. Transactions of the ASAE, v. 39, n. 6, p. 2007-16, 1996.

COELHO, E. F.; VELlAME, L. M.; COELHO FILHO, M. A.; LEDO, C. A. S. Desempenho de modelos de calibração de guias de onda acopladas a TDR e a multiplexadores em três tipos de solos. Revista Brasileira de Ciência do Solo, v. 30, p 23-30, 2006.

DALTON, F. N. Development of time domain reflectometry for measuring soil water and bulk soil electrical conductivity. Soil Science Society American, v. 30, p. 143-167, 1992.

DAVIS, J. L.; ANNAN, A. P. Electromagnetic detection of soil moisture. Progress Report, 1. Can. J. Remote Sensing, v. 3, n. 1, p. 76-86, 1977.

DAVIS, J. L.; CHUDOBIAK, W. J. In situ meter for measuring relative permittivity of soils. Geol. Surv. Can., v. 75, p. 75-79, 1975. 
DE ASSIS, C. D. Avaliação de uma sonda TDR helicoidal para a estimativa do teor de umidade dos solos em campo e laboratório. 2008. 180f. Dissertação (Mestrado em Geotecnia) - Escola de Engenharia de São Carlos, Universidade de São Paulo, São Carlos, 2008.

GIESE, K.; TIEMANN, R. Determination of the complex permittivity from a thin sample time domain reflectometry, improved analysis of the step response waveform. Adv. Molec. Relax. Processes., v. 7, p. 45-49, 1975.

GOMES, E. R. S.; SAMPAIO, S. C.; CORRÊA, M. M.; VILAS BOAS, M. A.; ALVES, L. F. A.; ALVES SOBRINHO, T. Movimento de nitrato proveniente de água residuária em colunas de solos. Engenharia Agrícola, v. 24, n. 3, p. 557-568, 2004.

HEIMOVAARA, T. J.; FOCKE, A. G.; BOUTEN, W.; VERSTRANTEN, J. M. Assessing temporal variations in soil water composition with time domain reflectometry. Soil Science Society of America Journal, v. 59, p. 689-698, 1995.

LELIS NETO, J. A. Monitoramento de componentes químicos da vinhaça aplicados em diferentes tipos de solo. 2008. Dissertação (Mestrado em Agronomia) - Universidade de São Paulo, Escola Superior de Agricultura Luiz de Queiroz, Piracicaba, 2008.

MMOLAWA, K.; OR, D. Root zone solute dynamics under drip irrigation: a review. Plant and Soil, v. 222, n. 1/2, p. 163-90, 2000.

MOJID, M. A.; WYSEURE, G. C. L.; ROSE, D. A. Extension of the measurement range of electrical conductivity by time domain reflectometry. Hidrological Earth Systems Science, v. 1, p. 175-183, 1997.

MUALEN, Y.; FRIEDMAN, S. P. Theoretical prediction of electrical conductivity in satured and unsatured soil. Water Resources Research, v. 27, p. 2771-2777, 1991.

MUCHOVEJ, R. M. C.; RECHCIGL, J. E. Impacts of nitrogen fertilization of pastures and turfgrasses on water quality. In: LAL, R.; STEWART, B. A. (Eds.). Soil processes and water quality. Boca Raton: Lewis Publication, 1994. p. 91-135.

NADLER, A.; FRENKEL, H.; MANTELL, A. Applicability of the four-probe technique under extremely variable water contents and salinity distribution. Soil Science Society of America Journal, v. 48, p. 1258-1261, 1984.

NADLER A.; DASBERG, S.; LAPID, I. Time domain reflectometry measurements of water content and electrical conductivity of layered soils columns. Soil Science Society America Journal, v. 55, p. 938-943, 1991.

OLIVEIRA, J. R. A.; VILELA, L.; AYARZA, M. A. Adsorção de nitrato em solos de cerrado do Distrito Federal. Pesquisa Agropecuária Brasileira, v. 35, n. 6, p. 1199-1205, 2000.

OLIVEIRA, M. V. A. M. DE; VILLAS BÔAS, R. L. Uniformidade de distribuição do potássio e do nitrogênio em sistema de irrigação por gotejamento. Engenharia Agrícola, v. 28, n. 1, p. 95-103, 2008.

PERSSON, M.; UVO, C. B. Estimating soil solution electrical conductivity from time domain reflectometry measurements using neural networks. Journal of Hydrology, v. 273, n. 1/4, p. 249-256, 2003. 
REICHARD, K.; TIMM, L. C. Solo, planta e atmosfera: conceitos, processos e aplicações. Barueri: Manole, 2004. 478 p.

RHOADES, J. D.; RAATS, P. A.; PRATHER, R. J. Effects of liquid phase electrical conductivity, water content, and surface conductivity on bulk soil electrical conductivity. Soil Science Society of America Journal, v. 40, p. 651-655, 1976.

RHOADES, J. D.; MANTEGHI, N. A.; SHOUSE, P. J.; ALVES, W. J. Soil electrical conductivity and salinity: new formulations and calibrations. Soil Science Society America Journal, v. 53, p. 433-439, 1989.

RIBEIRO, A. C.; GUIMARÃES, P. T. G.; ALVAREZ, V. V. H. Recomendações para o uso de corretivos e fertilizantes em Minas Gerais. Viçosa: Comissão de fertilidade do solo do Estado de Minas Gerais, 1999. 359p

ROLIM NETO, F. C. Gênese, química, mineralogia e micromorfologia de topolitossequências de solos do Alto Paranaíba - MG. 2002. 143f. Tese (Doutorado em Solos) - Universidade Federal de Viçosa, Viçosa, 2002.

SANTANA, G. S.; COELHO, E. F.; SILVA, T. S. M.; RAMOS, M. M. Relação entre potássio na solução do solo, umidade e condutividade elétrica aparente do solo. Revista Brasileira de Engenharia Agrícola e Ambiental, v. 11, n. 2, p. 142-157, 2007.

SANTOS, M. R. Uso da reflectometria no domínio do tempo para avaliar a distribuição de nitrato e fósforo em colunas de solos fertirrigados. 2008. 59f. Dissertação (Mestrado em Engenharia Agrícola) - Universidade Federal de Viçosa, Viçosa, 2008.

SHEN, F. Estimation of soil water and resolution and resident and effluent solute concentrations using time domain reflectometry. 1999. Dissertação (Mestrado em Engenharia Agrícola) - Iowa State University, Iowa, 1999.

SOUZA, C. F.; FOleGATTI, M. V.; MATSURA, E. E.; OR, D. Calibração da Reflectometria no Domínio do Tempo (TDR) para a estimativa da concentração da solução no solo. Engenharia Agrícola, v. 26, n. 1, p. 282-291, 2006.

SOUZA, T. V.; PAZ, V. P. S.; COELHO, E. F.; PEREIRA, F. A. C.; LEDO, C. A. S. Crescimento e produtividade do mamoeiro fertirrigado com diferentes combinações de fontes nitrogenadas. Irriga, v. 12, n. 4, p. 563-574, 2007a.

SOUZA, C. F.; FAVARO, R. B. T. M.; FOLEGATTI, M. V.; DAROZ, T. H. C.; FRANÇA E SILVA, E. F. Uso da reflectometria no domínio do tempo (TDR) para avaliar a distribuição do nitrato no volume molhado do solo sob fertirrigação. Magistra, v. 19, n. 2, p. 112-122, 2007b.

TOPP, G. C.; DAVIS, J. L.; ANNAN, A. P. Electromagnetic determination of soil water content:measurements in coaxial transmission lines. Water resources Research, v. 16, n. 3, p. 574-82, 1980.

TOPP, G. C.; YANUKA, M.; ZEBCHUK, W.D.; ZEELIN, S. Determination of electrical conductivity using time domain reflectometry: Soil and water experiments in coaxial lines. Water Resources Research, v. 24, p. 945-952, 1988. 
TRINTINALHA, M. A.; GONÇALVES, A. C. A.; TORMENA, C. A.; COSTA, A. C. S.; FOLEGATTI, M. V.; FREITAS, P. S. L.; REZENDE, R. Comparação dos sistemas TDR e ECHO para medida de umidade, em um solo argiloso e em areia. Acta Scientiarum, v. 26, p. 353-360, 2004.

VILLWOCK, R.; TAVARES, M. H. F.; VILAS BOAS, M. A. Calibração de um equipamento TDR em condições de campo. Irriga, v. 9, p. 82-88, 2004.

VOGELER, I.; CLOTRIER, B. E.; GREEN, S. R.; SCOTTER, D. R.; TILLMAN, R. W. Characterizing water and solute movement by TDR and disk permeametry. Soil Science Society of America Journal, v. 60, n. 1, p. 5-12, 1996.

WARD, P. R.; DUNIN, F. X. Growing season evapotranspiration from duplex soils in southwestern Australia. Agricultural Water Management, v. 50, n. 2, p. 141-159, 2001.

$\mathrm{YU}, \mathrm{C}$. The application of time domain reflectometry in solute transport experiments. 1998. Dissertação (Mestrado em Hidrologia) - Departamento de Hidrologia e Recursos Aquáticos da Universidade do Arizona, Arizona, 1998. 